\title{
Zurek-Kibble Symmetry Breaking Process in Superconducting Rings; Spontaneous Fluxon Formation in Annular Josephson Tunnel Junctions
}

\author{
Aarøe, Morten; Monaco, Roberto; Dmitriev, P; Koshelets, V. P.; Rivers, R. J.; Mygind, Jesper
}

Published in:

I E E E Transactions on Applied Superconductivity

Link to article, DOI:

10.1109/TASC.2007.898054

Publication date:

2007

Document Version

Publisher's PDF, also known as Version of record

Link back to DTU Orbit

Citation (APA):

Aarøe, M., Monaco, R., Dmitriev, P., Koshelets, V. P., Rivers, R. J., \& Mygind, J. (2007). Zurek-Kibble Symmetry Breaking Process in Superconducting Rings; Spontaneous Fluxon Formation in Annular Josephson Tunnel Junctions. I E E E Transactions on Applied Superconductivity, 17(2), 664 - 667. https://doi.org/10.1109/TASC.2007.898054

\section{General rights}

Copyright and moral rights for the publications made accessible in the public portal are retained by the authors and/or other copyright owners and it is a condition of accessing publications that users recognise and abide by the legal requirements associated with these rights.

- Users may download and print one copy of any publication from the public portal for the purpose of private study or research.

- You may not further distribute the material or use it for any profit-making activity or commercial gain

- You may freely distribute the URL identifying the publication in the public portal 


\title{
Zurek-Kibble Symmetry Breaking Process in Superconducting Rings; Spontaneous Fluxon Formation in Annular Josephson Tunnel Junctions
}

\author{
M. Aaroe, R. Monaco, P. Dmitriev, V. P. Koshelets, R. J. Rivers, and J. Mygind
}

\begin{abstract}
We report on new investigations of spontaneous symmetry breaking in non-adiabatic phase transitions. This Zurek-Kibble (ZK) process is mimicked in solid state systems by trapping of magnetic flux quanta, fluxons, in a long annular Josephson tunnel junction quenched through the normal-superconducting transition. A trapped fluxon unambiguously is detected as a zero-field step in the DC I-V characteristic. Experimentally we plot the fluxon trapping probability versus the quench rate, varied over 4 decades. An allometric scaling behavior is found. By fitting to the theoretical curve we get $\sigma \simeq 0.5$ for the $\mathrm{ZK}$ critical scaling exponent $\sigma$, which does not agree with an earlier theoretical prediction of $\sigma=0.25$. A novel theory based on the proximity effect leading to $\sigma=0.50$ has been proposed. The dependence of the gap voltage on temperature is measured and used for precise monitoring of the fast temperature variation during the quench.
\end{abstract}

\section{INTRODUCTION}

C AUSALITY is ubiquitous, both in the early universe, where the propagation of field fluctuations is constrained by the speed of light, and in condensed matter, now constrained by the speed of sound. Although, under adiabatic change, correlation lengths do diverge at the critical temperature $T_{C}$, in reality causality prevents any lengths diverging since transitions take place in a finite time. The proposal that the domain structure after a transition is determined by causality, in the sense that the resulting domain structure reflects causal horizons, was first made by Kibble [1] for the early universe and, independently, by Zurek [2], [3] for condensed matter systems. In particular, if transitions are frustrated, topological defects arise to mediate the different ground states. The defect density will be related to the nature of the domain structure present and

Manuscript received August 28, 2006. We thank A. Sobolev and M. Torgashin for sample fabrication and U.L.Olsen for help at the initial stage of the experiment. This work is, in part, supported by the COSLAB programme of the European Science Foundation, the Danish Research Council, the Hartmann Foundation, the RFBR project 06-02-17206, and the Grant for Leading Scientific School 7812.2006.2.

M. Aaroe and J. Mygind are with the Department of Physics, B309, Technical University of Denmark, DK-2800 Lyngby, Denmark (e-mail: myg @fysik.dtu. $\mathrm{dk})$.

R. Monaco is with Istituto di Cibernetica del C.N.R., 80078, Pozzuoli, Italy, and also with Unita' INFM-Dipartimento di Fisica, Universita' di Salerno, 84081 Baronissi, Italy (e-mail: roberto@sa.infn.it).

P. Dmitriev and V. P. Koshelets are with the Institute of Radio Engineering and Electronics, Russian Academy of Science, Mokhovaya 11, 125009, Moscow, Russia (e-mail: valery@ @itech.cplire.ru).

R. J. Rivers is with Blackett Laboratory, Imperial College London, London SW7 2AZ, U.K. (e-mail: r.rivers@imperial.ac.uk).

Digital Object Identifier 10.1109/TASC.2007.898054 be constrained by causality in turn, so counting the defects, in principle, permits us to check the ZK proposition. The aim of the experiments discussed below [4]-[6] is to test this scenario for continuous phase transitions in superconductors.

The ZK picture predicts a characteristic scaling behavior in the quench time $\tau_{Q}$ (the inverse quench rate) defined by

$$
\frac{T_{C}}{\tau_{Q}}=-\left.\frac{d T}{d t}\right|_{T=T_{C}} .
$$

Specifically, if $\bar{\xi}$ is the separation of defects at the time of their production then it scales with $\tau_{Q}$ as

$$
\bar{\xi} \approx \xi_{0}\left(\frac{\tau_{Q}}{\tau_{0}}\right)^{\sigma} \text {. }
$$

where $\xi_{0}$ is, most simply, the cold correlation length, $\tau_{0}$ the relaxation time of the long wavelength modes, and $\sigma>0$ is the scaling exponent.

For long annular Josephson tunnel junctions (AJTJs) the topological defect is a fluxon i.e. a super current vortex carrying a single quantum of magnetic flux $\Phi_{0}=h /(2 e)$ in the plane of the oxide layer between the two superconducting rings that make up the junction. While Josephson junctions possess the virtues of superconductors, they avoid many of the problems encountered by other experiments performed to test the ZK picture that are commensurate with it or, when not, explicable in its framework [7]-[15]. Our experiments are the only ones with condensed matter systems to date that are sensitive enough to show unambiguous scaling behavior.

However, we do have a potential problem in that (2), couched in the language of causal horizons, is not designed for systems, such as ours, that are much smaller than them. Instead, we propose that the probability $f_{1}$ for spontaneously producing one fluxon in the thermal quench of a symmetric AJTJ of circumference $C<\bar{\xi}$ should scale with the quench time $\tau_{Q}$ as

$$
f_{1} \simeq \frac{C}{\bar{\xi}}=\frac{C}{\xi_{0}}\left(\frac{\tau_{Q}}{\tau_{0}}\right)^{-\sigma},
$$

where $\sigma$ depends on the nature of the junction. Since our samples are relatively small $C<\bar{\xi}$ we only expect to see few trapped fluxons in each measurement, at best. Besides, because AJTJs act as their own thermometers, $\tau_{Q}$ can be measured to high accuracy (see below).

To derive $\sigma$ two of us had argued earlier [16], [17] that the relevant causality is provided by the finite velocity of electromagnetic waves in the JTJ, the Swihart velocity. Under the idealistic assumptions of a) weak coupling of the superconductors 


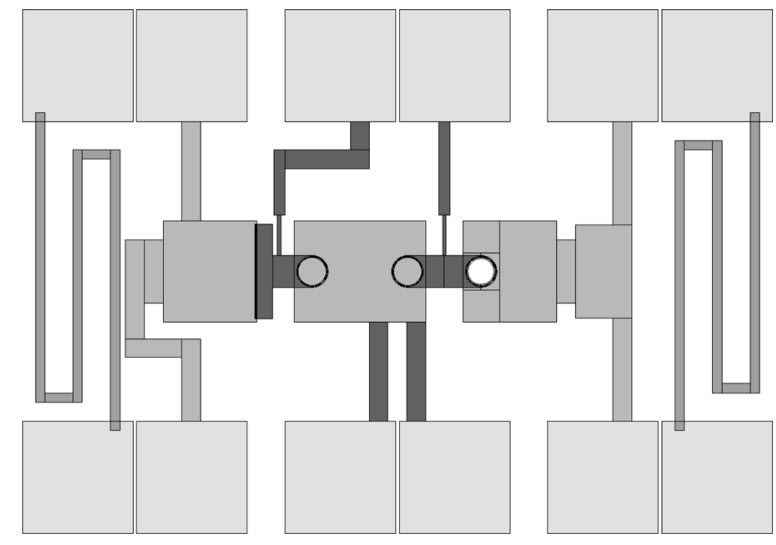

Fig. 1. Layout of the $4.2 \mathrm{~mm} \times 3 \mathrm{~mm} \mathrm{Si}$ chip containing four series biased $\mathrm{Nb}-\mathrm{Al} / \mathrm{Al}_{o x} / \mathrm{Nb}$ Josephson tunnel junctions. It integrates one $4 \times 500 \mu \mathrm{m}^{2}$ overlap-type linear junction (\#1), three ring shaped junctions (\#2,\#3,\#4) having a mean circumference $\mathrm{C}=500 \mu \mathrm{m}$ and a width $\Delta \mathrm{r}=4 \mu \mathrm{m}$, and two meanderline resistive Mo strips used for heating.

and b) exact critical slowing of the Swihart velocity at the critical temperature $T=T_{C}$, we predicted [16], [17] $\sigma=0.25$. The experiment was successful, with $\sigma$ commensurate with scaling behavior (3) with this value of $\sigma$.

The new experiments, described in this paper, have forced us to revise our assumptions. In our recent publication [6] we showed new scaling behavior for the spontaneous production of fluxons, in which $f_{1}$ was indeed seen to clearly follow an allometric dependence on $\tau_{Q}$, but with a scaling exponent $\sigma=0.5$. We discuss this experiment below and confirm this behavior with data from new samples (see Fig. 3). The discrepancy between the observations of the early and late experimental values of $\sigma$ may be less than it looks at first sight, given the high accuracy of the latter and the relative scatter of the former.

\section{SAMPLES AND SETUP}

We use high quality $\mathrm{Nb} / \mathrm{Al}-\mathrm{Al}_{o x} / \mathrm{Nb}$ AJTJs fabricated on a silicon substrate with the trilayer technique in which the junction is realized in a "window" opened in the $\mathrm{SiO}$ insulator layer. The so called "idle region", is about $3 \mu \mathrm{m}$. The thicknesses of the base, top and wiring layer are 200, 80 and 400nm, respectively. Details of the fabrication process can be found in [18]. All the experiments performed to date have been carried out on AJTJs with a circumference $\mathrm{C}=500 \mu \mathrm{m}$ and a width $\Delta \mathrm{r}=4 \mu \mathrm{m}$. AJTJs with larger circumferences have been fabricated, but we have yet to use them.

The chip layout is shown in Fig. 1. It integrates three AJTJs and one $4 \times 500 \mu \mathrm{m}^{2}$ overlap-type linear junction (\#1). The rightmost AJTJ (\#4) is obtained by the superposition of two superconducting rings, while the two AJTJs (\#2 and \#3) in the layout middle are realized by the superposition of a ring shaped top electrode over a superconducting ground plane. The four junctions (numbered from left) are current biased in series.

For the new experiment a faster and more reliable heating system was required. This is achieved by integrating a meander line $50 \mu \mathrm{m}$ wide and $200 \mathrm{~nm}$ thick Mo resistive film in either ends of the $4.2 \mathrm{~mm} \times 3 \mathrm{~mm} \times 0.35 \mathrm{~mm}$ Si chip. Each resistive element has a DC resistance of $50 \Omega$ at LHe temperatures. In fact, voltage pulses a few $\mu$ s long and a few volts high applied across the integrated heater provide quench times as low as $1 \mathrm{~ms}$, two orders of magnitude smaller than with the previous layout [4], [5].

A detailed description of the experimental setup has been given in [5]. Briefly, the chip with the AJTJs is mounted onto a $\mathrm{Cu}$ block enclosed in a vacuum-tight can immersed in the liquid $\mathrm{He}$ bath. A Ge thermometer anchored to the Cu block allows for precise measurements of the chip temperature, when it is static or slowly changing. The junction itself is used for measurement of quickly changing temperatures (see below). The chip is heated above the critical temperature of the AJTJ by a voltage pulse applied to both meander line heaters. After the pulse the heat leaves the chip both through the thermal contact with the $\mathrm{Cu}$ block and by the $\mathrm{He}$ exchange gas inside the can. The He exchange gas pressure was fixed at a value of about $7 \mathrm{mbar}$. Particular care was taken to minimize thermal gradients (see below).

During the transition all electrical connections are disconnected from the chip, thermometers, etc. At the end of each cooling cycle a spontaneously generated fluxons will be trapped in the AJTJ, and remain static. After a while an external current is supplied to the AJTJ. The current sets the fluxon (if any) in motion around the annulus and quantized voltages (so-called Zero Field Steps, ZFSs) develop across the junction. In other words, we can count the number of produced fluxons (defects) by a careful inspection of the junction DC current-voltage characteristic (IVC). Due to small junction losses the fluxon motion is rather unstable at $4.2 \mathrm{~K}$, therefore the IVC is better inspected at higher temperatures $(6-7 \mathrm{~K})$ where larger losses stabilize the fluxon motion.

Great efforts have been devoted to reduce spurious DC and AC magnetic fields. Only non-magnetic materials were used in the vicinity of the chip. We used a double $\mu$-metal shielded cryostat and the sample holder vacuum can was surrounded by a multi-layer thin superconducting $\mathrm{Pb}$ shield. In turn the chip holder inside the can was enclosed in a cryoperm shield and in one more superconducting $\mathrm{Pb}$ shield. Finally, all the measurements were carried out in an electromagnetically shielded room.

In order to run batches of several thousand equal thermal cycles with given parameters, automatization of thermal cycles was implemented by means of a switching unit controlled by a GPIB interface; that also allowed for much more robust statistics to be achieved. At the end of each thermal quench the junction IVC is automatically digitally acquired, converted and stored. Then at the end of each batch with a given value of $\tau_{Q}$ an algorithm has been developed for the analysis of the large amount of IVCs and the automatic detection and counting of the spontaneously trapped fluxons.

\section{Determining the Quenching Time}

The quench time $\tau_{Q}$ was varied over more that four orders of magnitude (from 20s down to $1 \mathrm{~ms}$ ) by varying the width and the amplitude of the voltage pulse across the integrated resistive elements. In order to estimate the quenching time $\tau_{Q}$ we use the junction itself as a thermometer. The temperature dependence of 


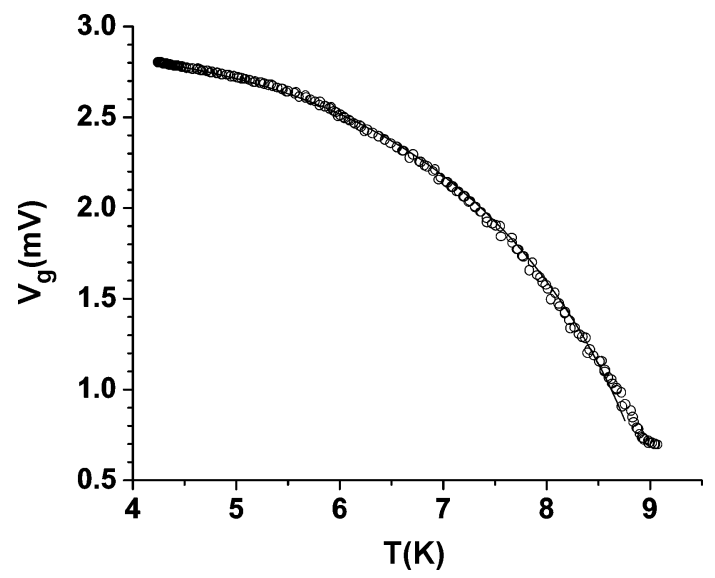

Fig. 2. The temperature dependence of the gap voltage $V_{g}$. The open circles are the experimental data with the junction biased at $25 \%$ of the total current jump at the gap voltage; the solid line is the prediction that follows from (4). The fit yields $T_{C}=(9.12 \pm 0.04) \mathrm{K}$ and $V_{g}(T=0)=(2.89 \pm 0.02) \mathrm{mV}$.

the gap energy $\Delta(T)$ in a strong-coupling superconductor [19], such as $\mathrm{Nb}$,

$$
\frac{\Delta(T)}{\Delta(0)}=\tanh \frac{\Delta(T)}{\Delta(0)} \frac{T_{C}}{T},
$$

also applies to the gap voltage $V_{g}$ that is proportional to $\Delta$.

In our samples we have found that, provided the AJTJ is current biased at about $20-25 \%$ of the total current jump at $V_{g}$, the junction temperature could be monitored to a high degree of accuracy and speed by resorting to (4). This is shown in Fig. 2 where the experimental values (open circles) of the $V_{g}(T)=$ $2 \Delta(T) / e$ are plotted versus temperature as measured by a calibrated Ge thermometer. The solid line is the prediction that follows from (4) with fitting parameters $T_{C}=(9.12 \pm 0.04) \mathrm{K}$ and $V_{g}(0)=(2.89 \pm 0.02) \mathrm{mV}$, i.e. $2 \Delta(0) / k_{B} T_{c} \simeq 3.73 \pm 0.03$. As a result, (4) can be used to conveniently estimate the junction temperature for $V_{g}(T)<1 \mathrm{mV}$, i.e., $T<8.5 \mathrm{~K}$. Above $8.5 \mathrm{~K}$ the experimental data for $V_{g}$ saturate to a temperature-independent value corresponding to the product of the normal resistance and the bias current. However, the overall temperature accuracy $\delta T=\left|\left(d T / d V_{g}\right)\right| \delta V_{g}$ cannot be smaller than $2 \mathrm{mK}$ due to limited sensitivity $\delta V_{g} \approx 2 \mu \mathrm{V}$ of the digital oscilloscope.

Assuming the chip exchanges heat mainly through a massive copper base plate (with thermal constant $\tau_{1}$ ) which in turn exchanges heat with the surrounding helium gas (with thermal constant $\tau_{2}$ ), the thermal relaxation during the thermal quench has been fitted by a double exponential decay of the form

$T(t)=T_{f i n}+\Delta T_{1} \exp \left(-\frac{t-t_{0}}{\tau_{1}}\right)+\Delta T_{2} \exp \left(-\frac{t-t_{0}}{\tau_{2}}\right)$,

with $\Delta T_{1}, \Delta T_{2}, \tau_{1}$ and $\tau_{2}$ as fitting parameters. $T_{f i n}$ and $t_{0}$ are known from the experiments. If the time origin is triggered by the voltage pulse, then $t_{0}$ corresponds to the time at which the pulse ends. Once the parameters in (5) are determined or measured, the quenching time $\tau_{Q}$ can be inferred from its definition (1), after (5) has been extrapolated up to the critical temperature $T_{C}$. At the end of this process of fitting and extrapolation, $\tau_{Q}$ is known to an overall accuracy of about $\pm 10 \%$.

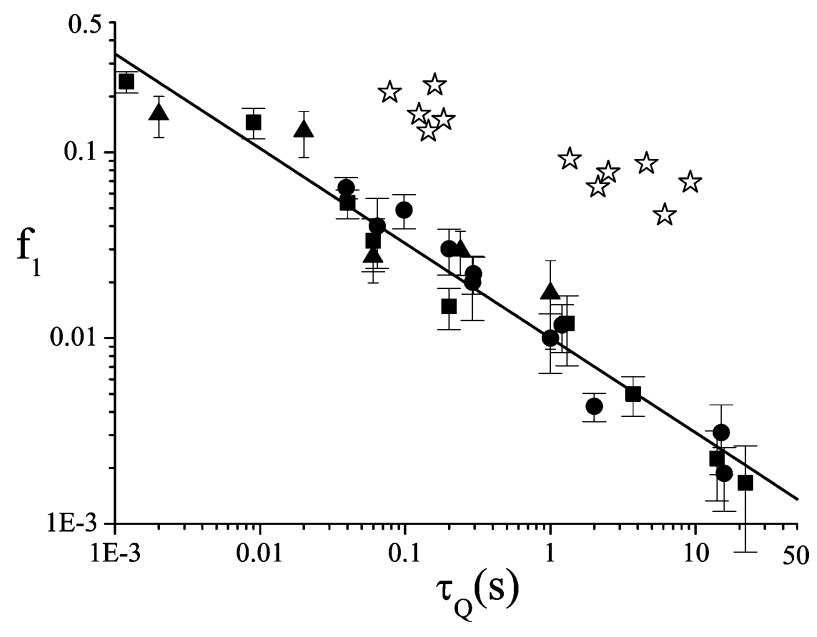

Fig. 3. Log-log plot of the frequency $f_{1}$ of trapping single fluxons versus the quenching time $\tau_{Q}$. Each point corresponds to many thermal cycles, closed squares for sample 22-4, closed triangles for sample 08-3 and closed circles for sample 08-4. The vertical error bars gives the statistical error while the relative error bars $( \pm 10 \%)$ in $\tau_{Q}$ are as large as the dots' width. The solid line is the fit of all data to an allometric relationship $f_{1}=a \tau_{Q}^{-b}$ which yields $a=0.01 \pm 10 \%$ (taking $\tau_{Q}$ in seconds) and $b=0.51 \pm 5 \%$. The open stars represent the data obtained in a previous experiment [4], [5].

\section{THE ZK MEASUREMENTS}

The experimental results reported here refer to three identical AJTJs belonging to two different chips made within the same batch having a critical current density $J_{c}(0) \simeq 60 \mathrm{~A} / \mathrm{cm}^{2}$ yielding a Josephson penetration depth $\lambda_{J}(0) \simeq 50 \mu \mathrm{m}$. The assumption of strong coupling junction electrodes leads to a value of $\xi_{0} \simeq 17 \mu \mathrm{m} \mathrm{[6].} \mathrm{For} \mathrm{all} \mathrm{samples} \mathrm{the} \mathrm{high} \mathrm{quality} \mathrm{has} \mathrm{been}$ inferred by a measure of the IVC at $T=4.2 \mathrm{~K}$. Due to the very high reliability of the fabrication line the AJTJs have the same geometrical and electrical parameters. However, to distinguish them we will name them after their chip and junction numbers; 08-\#3, 08-\#4 and 22-\#4 (see caption to Fig. 1). No other junctions have been tested. Samples 08-3 and 08-4 belonged to the same chip. As depicted in Fig. 1 the bottom electrodes of the AJTJs 08-4 and 22-4 were hollow while for 08-03 the bottom electrode formed a superconducting ground plane.

The solid symbols in Fig. 3 show on a log-log plot the measured frequency $f_{1}=n_{1} / N$ of single fluxon trapping, obtained by quenching the samples $\mathrm{N}$ times for each value of a given quenching time $\tau_{Q}, n_{1}$ being the number of times that the inspection of the IVC at the end of each thermal cycle showed that a fluxon was trapped. $\mathrm{N}$ ranged between 100 and 5000 and $n_{1}$ was never smaller then 10 , except for some of the rightmost points $\left(\tau_{Q}<10 \mathrm{~s}\right)$ for which $n_{1} \geq 3$. All samples has undergone a total of more than 100,000 thermal cycles without any measurable change of their electrical parameters. The vertical error bars gives the statistical error $f_{1} / \sqrt{ } n_{1}$. The relative error bars in $\tau_{Q}$ amounting to $\pm 10 \%$ are as large as the dot's width.

It is quite evident that the dependence of the trapping frequency on the quenching time is the same for all three AJTJs independent on the geometry of their base electrodes. Careful measurements of the junction IVC during the N-S transition indicate that the junction critical temperature might differ from the critical temperature of the base electrode film by no more than 
$10 \mathrm{mK}$, while the critical temperature of the wiring film, being twice thicker, exceeded the critical temperature of the base electrode by about $100 \mathrm{mK}$. In other words, at the time the Josephson effect is installed, the base electrode is only weakly superconducting and cannot exercise any shielding effect. This explains why we have not observed any difference between the data of junction 08-3 and those of junctions 08-4 and 22-4.

To test (3), we have fitted the data of all samples with the same allometric function $f_{1}=a \tau_{Q}^{-b}$, with $a$ and $b$ as free fitting parameters. A linear regression of $\log f_{1}$ vs. $\log \tau_{Q}$, represented by the continuous line in Fig. 3, yields $a=0.01 \pm 10 \%$ (taking $\tau_{Q}$ in seconds) and $b=0.51 \pm 5 \%$. Therefore the present experiment suggests that the scaling exponent is $\sigma=0.5$, rather than the value $\sigma=0.25$ suggested by our earlier attempt [4], [5]. One possible explanation [5], [6] lies in the fact that our strongly coupled AJTJs are far from ideal, showing i) a proximity effect that makes the critical current vanish faster at $T_{c}$ and ii) the Swihart velocity does not show exact critical slowing down. Taken together, these imply $\sigma=0.5$. On no occasion were two fluxons seen. Since we cannot detect a fluxon-antifluxon pair, this suggests a strong correlation between fluxon and antifluxon creation, although we have no compelling arguments for this.

For comparison, the data of the earlier experiment are reported in Fig. 3 as open stars. The shift in intercept (or, equivalently, prefactor $a$ ) between the two sets of data is to be expected. The AJTJs used in the first experiment, although of the same geometry as samples 08-4 and 22-4, had a Josephson current density $J_{c}$ about 60 times larger. This means a smaller Zurek length $\bar{\xi} \propto 1 / \sqrt{J}_{c}$, with a correspondingly greater likelihood of observing a fluxon. Even then, the value $a=0.01 \pm 10 \%$ (taking $\tau_{Q}$ in seconds) is 6-7 times larger than the predicted value. As a bound we only expect agreement in the overall normalization $a$ to somewhat better than an order of magnitude. Empirically, the different condensed matter experiments have shown that the ratio $a_{\text {observed }} / a_{\text {predicted }}$ varies widely from system to system.

The trapping frequency depends on the magnetic field applied to the chip. Although the absolute value of the DC residual magnetic field cannot be measured, we have checked that $f_{1}$ did not change i) by rotating the cryoprobe in the horizontal plane (this changes the sample orientation with respect to the direction of the earth's magnetic field) and ii) by rotating the chip holder inside the cryoprobe (this changes the sample orientation with respect to the shields). Furthermore, detailed measurements have been carried out of the dependence of the trapping frequency on the strength of an external field applied perpendicular to the junction plane for different samples and for several values of the quenching time. Such data will be reported in another paper [20]. As far as the measurements presented in the present work we adjusted the external magnetic field so that for each value of the quenching time the corresponding trapping frequency lies at the minimum.

\section{CONCLUSIONS}

Equation (3) is amenable to further experimental tests with AJTJs having different critical current densities $J_{c}(0)$ and/or circumferences $C$. According to theory [6], [20] $f_{1}$ scales as the fourth root of $J_{c}(0)$, and depends linearly on $C$ (see (3)). Also experiments with asymmetric $\mathrm{Nb}-\mathrm{AlN}-\mathrm{NbN}$ junctions for which we would expect a smaller value for $\sigma$ are in process of being performed. It is worth commenting on the effect of the unavoidable thermal gradients in physical system undergoing a thermal quench; the Zurek-Kibble scaling law (3) was derived assuming that thermal gradients are not a problem. We believe that thermal gradients are small (less than 10mK) in our chips, but this has to be proven in future experiments.

In summary, we see this experiment as providing unambiguous corroboration of Zurek-Kibble scaling over a wide range of quenching time $\tau_{Q}$ in accord with our predictions for $\mathrm{Nb}-\mathrm{Al} / \mathrm{Al}_{O x} / \mathrm{Nb}$ JTJs. As such, it replaces our less sophisticated earlier experiment [4], [5].

\section{REFERENCES}

[1] T. W. B. Kibble, J. Phys., vol. A 9, p. 1387, 1976.

[2] W. H. Zurek, Nature, vol. 317, p. 505, 1985.

[3] W. H. Zurek, Acta Physica Polonica, vol. B24, p. 1301, 1993.

[4] R. Monaco, J. Mygind, and R. J. Rivers, Phys. Rev. Lett., vol. 89, p. 080603, 2002.

[5] R. Monaco, J. Mygind, and R. J. Rivers, Phys. Rev., vol. B67, p. 104506, 2003.

[6] R. Monaco, J. Mygind, M. Aaroe, V. P. Koshelets, and R. J. Rivers, Phys. Rev. Lett., vol. 96, p. 180604, 2006.

[7] I. Chuang, R. Durrer, N. Turok, and B. Yurke, Science, vol. 251, p. 1336,1991

[8] S. Digal, R. Ray, and A. M. Srivastava, Phys. Rev. Lett, vol. 83, p. 5030, 1999.

[9] M. E. Dodd et al., Phys. Rev. Lett., vol. 81, p. 3703, 1998.

[10] M. E. Dodd et al., J. Low Temp. Physics, vol. 15, p. 89, 1999.

[11] C. Bauerle et al., Nature, vol. 382, p. 332, 1996.

[12] V. M. H. Ruutu et al., Nature, vol. 382, p. 334, 1996.

[13] A. Maniv, E. Polturak, and G. Koren, Phys. Rev. Lett., vol. 91, p. 197001, 2003.

[14] J. R. Kirtley, C. C. Tsuei, and F. Tafuri, Phys. Rev. Lett., vol. 90, p. 257001, 2003.

[15] S. Casado, W. González-Viñas, H. Mancini, and S. Boccaletti, Phys. Rev., vol. E63, p. 057301, 2001.

[16] E. Kavoussanaki, R. Monaco, and R. J. Rivers, Phys. Rev. Lett., vol. 85 , p. 3452, 2000.

[17] R. Monaco, R. J. Rivers, and E. Kavoussanaki, Journal of Low Temperature Physics, vol. 124, p. 85, 2001.

[18] P. N. Dmitriev, I. L. Lapitskaya, L. V. Filippenko, A. B. Ermakov, S. V. Shitov, G. V. Prokopenko, S. A. Kovtonyuk, and V. P. Koshelets, IEEE Trans. Appl. Supercond, vol. 13, pp. 107-110, 2003.

[19] R. F. Broom, J. Appl. Phys., vol. 47, p. 5432, 1976.

[20] R. Monaco, J. Mygind, M. Aaroe, V. P. Koshelets, and R. J. Rivers, in preparation. 